\title{
New field-in-field with two reference points method for whole breast radiotherapy: Dosimetric analysis and radiation-induced skin toxicities assessment
}

\author{
NOUHA TEKIKI $^{1}$, MASAHIRO KURODA ${ }^{2}$, HINATA ISHIZAKA ${ }^{2}$, ABDULLAH KHASAWNEH $^{1}$, \\ MAJD BARHAM $^{1}$, KENTARO HAMADA ${ }^{2}$, KOHEI KONISHI ${ }^{2}$, KOHEI SUGIMOTO ${ }^{2}$, KUNIAKI KATSUI ${ }^{3}$, \\ SOICHI SUGIYAMA ${ }^{4}$, KENTA WATANABE ${ }^{4}$, KOTARO YOSHIO $^{4}$, NORIHISA KATAYAMA ${ }^{5}$, \\ TAKESHI OGATA $^{6}$, HIROKI IHARA ${ }^{7}$, SUSUMU KANAZAWA $^{8}$ and JUNICHI ASAUMI ${ }^{1}$

\begin{abstract}
${ }^{1}$ Department of Oral and Maxillofacial Radiology, Okayama University Graduate School of Medicine, Dentistry and Pharmaceutical Sciences; ${ }^{2}$ Department of Radiological Technology, Graduate School of Health Sciences, Okayama University; ${ }^{3}$ Department of Proton Beam Therapy, Okayama University Graduate School of Medicine,

Dentistry and Pharmaceutical Science; ${ }^{4}$ Department of Radiology, Okayama University Hospital, Okayama 700-8558; ${ }^{5}$ Department of Radiology, Kagawa Prefectural Central Hospital, Takamatsu, Kagawa 760-8557;

${ }^{6}$ Department of Radiology, Iwakuni Clinical Center, Iwakuni, Yamaguchi 740-8510; ${ }^{7}$ Department of Radiology,

Tsuyama Chuo Hospital, Okayama $708-0841 ;{ }^{8}$ Department of Radiology, Okayama University

Graduate School of Medicine, Dentistry and Pharmaceutical Sciences, Okayama 700-8558, Japan
\end{abstract}

Received July 24, 2020; Accepted June 16, 2021

DOI: $10.3892 / \mathrm{mco} .2021 .2355$

\begin{abstract}
The usefulness of the field-in-field with two reference points (FIF w/2RP) method, in which the dose reference points are set simultaneously at two positions in the irradiation field and the high-dose range is completely eliminated, was examined in the present study with the aim of decreasing acute skin toxicity in adjuvant breast radiotherapy (RT). A total of 573 patients with breast cancer who underwent postoperative whole breast RT were classified into 178 cases with wedge (W) method, 142 cases with field-in-field without 2 reference points (FIF w/o 2RP) method and 253 cases with FIF w/ 2RP method. Using the FIF w/ 2RP method, the high-dose range was the lowest among the three irradiation methods. The planning target volume (PTV) V105\% and the breast PTV for evaluation (BPe) V105\% decreased to 0.09 and $0.10 \%$, respectively. The FIF w/ 2RP method vs. the FIF w/o 2RP method had a strong association $(\eta)$ with PTV V105\% $(\eta=0.79 ; P<0.001)$ and BPe V105\% $(\eta=0.76 ; P<0.001)$. The FIF w/ 2RP method had a significant impact on lowering the skin toxicity grade in weeks 3 and 4 , and increasing the occurrence
\end{abstract}

Correspondence to: Professor Masahiro Kuroda, Department of Radiological Technology, Graduate School of Health Sciences, Okayama University, 2-5-1 Shikata-cho, Okayama 700-8558, Japan

E-mail: kurodamd@cc.okayama-u.ac.jp

Key words: breast cancer, field-in-field radiotherapy, dose reference point, acute skin toxicity, dose distribution, high-dose area of skin toxicity grade 0 . The FIF w/ 2RP method vs. the $\mathrm{W}$ method had a moderate association with skin toxicity grade at week $3(\eta=0.49 ; \mathrm{P}<0.001)$. Using the FIF w/ 2RP method, the high-dose range $\mathrm{V} 105 \%$ of the target decreased to $0 \%$, and skin adverse events were decreased in conjunction. For patients with early-stage breast cancer, particularly patients with relatively small-sized breasts, the FIF w/ 2RP method may be an optimal irradiation method.

\section{Introduction}

For women with early-stage breast cancer, whole breast radiotherapy (WBRT) after breast-conserving surgery (BCS) is a longstanding standard practice. WBRT reduces the risk of locoregional and distant first recurrence by $50 \%$, and reduces breast cancer death by $\sim 1 / 6(1,2)$. However, most patients with breast cancer who undergo adjuvant radiotherapy (RT) are affected by skin toxicities (3). During RT treatment, these skin toxicities cause physical discomfort and emotional distress, and therefore negatively affect the patients' quality of life (4). In consequence, previous studies investigated factors associated with an increased risk of developing acute skin toxicities. Dose distribution and resulting dosimetric parameters (in particular high-dose ranges of $>105,>107$ and $>110 \%$ within the target volume) are RT treatment procedure-related factors, reported to be significantly associated with an increased risk of developing skin toxicities (5-8).

In modern RT, field-in-field (FIF) techniques are considered the standard-of-care when three-dimensional conformal RT (3D-CRT) treatment planning is available $(5,9)$. However, using the FIF for patients who have relatively small-sized breasts results in high-dose areas remaining in the irradiation 
field around the reference point. Therefore, in our institution, we implemented a new FIF technique, the field-in-field with 2 reference points (FIF w/ 2RP) method, aimed at improving dosimetric parameters and thus reducing high-dose ranges and subsequent skin toxicities.

The current study sought to examine the usefulness of this new irradiation method. We performed a comparative analysis of dosimetric parameters of three irradiation methods including the FIF w/ 2RP method and two other irradiation methods, the wedge (W) method and the field-in-field without 2 reference points (FIF w/o 2RP) method, as control. Furthermore, we assessed radiation-induced skin toxicities. We then investigated the association of each irradiation method and skin toxicities.

\section{Materials and methods}

Patients. Between April 2008 and December 2016, 577 females with early-stage breast cancer treated at Okayama University Hospital with BCS and adjuvant RT were evaluated. These patients were selected from 723 consecutive females with early-stage breast cancer. Exclusion criteria were simultaneous bilateral breast cancer, treatment with regional nodal irradiation and treatment using hypo-fractionated irradiation. Furthermore, four patients treated without the use of wedge filters or the FIF method were excluded from this study due to insufficient sample size. Finally, data regarding 573 patients, aged 24-82 years (mean, 55 years), were evaluated. Patients provided written informed consent for undergoing RT and using their anonymous data for scientific studies. This study was conducted in accordance with the Declaration of Helsinki, as revised in 2013.

Radiation treatment. Each patient received WBRT in 25 consecutive fractions of 2 Gy to a total prescribed dose of $50 \mathrm{~Gy}$. In total, 271 patients received a sequential boost of 10 or 16 Gy to the tumor bed. Free-breathing computed tomography (CT) acquisition was performed with an Asteion Super4 Edition multi-slice CT scanner (Toshiba Corporation) and reconstructed with $2 \mathrm{~mm}$-thick slices. To set the irradiation field, radio-opaque markers were placed on external landmarks; the sternal notch, the midline, the mid-axillary line, $1 \mathrm{~cm}$ below the infra-mammary fold, surgical scars, the nipple and the margin of palpable breast tissue; at the acquisition of the CT scan to facilitate contouring segmentation of the CT dataset. CT images were then transferred to the treatment planning system CMS Xio Ver 4.3.4 (Computerized Medical Systems, Inc.).

The planning target volume (PTV) was defined three-dimensionally as $5 \mathrm{~mm}$ inside the previously set irradiation field, excluding the part outside the patient and the first $5 \mathrm{~mm}$ of tissue under the skin, and posteriorly limited no deeper to the anterior surface of the ribs excluding the boney thorax and lung. Breast PTV Eval (BPe) was determined according to the clinical research protocol Radiation Therapy Oncology Group (RTOG) 1005 (10) and its defined clinical target volume (CTV) and PTV. This BPe volume was used for breast volume evaluation. Contoured organs at risk (OARs) were the lung and the heart. The heart and the axillary lymph nodes levels I, II and III were countered according to the RTOG-endorsed consensus guidelines for delineation of target and normal structures for breast cancer (11). These target contours were then checked and modified to be included within the previously set irradiation field to be 3D-CRT treatments. Beam energies were 4, 6 and $10 \mathrm{MV}$ by LINEACS (Mevatron M2/6327, PRIMUS High Energy KD2/7467 and ONCOR High Energy ONCR-K; Toshiba Corporation).

Three irradiation methods were performed: i) $\mathrm{W}$ method was used for 178 patients. The RP was set at the level of the nipple or at the mid-level between the upper and lower edges of the irradiation field. Physical wedges in the opposing tangential beams were used to improve target dose distribution. For some patients, to avoid hot spots, dose distribution was optimized by adding an extra tangent FIF; ii) the FIF w/o 2RP method was used for 142 patients. Two opposed tangential fields were set up without wedges. The RP was set at the mid-level between the upper and lower edges of the irradiation field or $2 \mathrm{~cm}$ apart from the deepest point and upper edge of the irradiation field. The main field was copied as subfields and the multileaf collimators (MLCs) were manipulated to shield the areas of the breast receiving a high dose. However, these MLCs were not allowed to block within $2 \mathrm{~cm}$ of the RP; and iii) the FIF w/ 2RP method was used for 253 patients. Two RPs were set for each patient; one RP for the main beam at a point $2 \mathrm{~cm}$ apart from the deepest point and upper edge of the irradiation field, and another RP for the FIF at the mid-level between the upper and lower edges of the irradiation field. As one RP was used in the FIF w/o 2RP method, the MLCs could not be inserted in the range of $2 \mathrm{~cm}$ around the $\mathrm{RP}$, which resulted in the high-dose area remaining close to that RP. For patients who have relatively small-sized breasts, this can be problematic, as it results in a high-dose area around that RP. Therefore, in the FIF w/ 2RP method, the RPs were set simultaneously at two places in the irradiation field, and high-dose areas were sequentially shielded and completely eliminated by the MLCs. Patients treated with the W method and the FIF w/o 2RP method were considered as control patients.

Dosimetric analysis. A dose distribution in the PTV and BPe of $95-107 \%$ according to ICRU criteria was obtained while lowering OARs doses as much as possible. The dosimetric parameters recorded for all plans, including PTV and BPe, were mean dose, $\mathrm{V} 0-95 \%$ (the volume percentage receiving less than $95 \%$ of the prescribed dose), V95-107\% (the volume percentage receiving between 95 and $107 \%$ of the prescribed dose), V107\% (the volume percentage receiving more than $107 \%$ of the prescribed dose) and V105\% (the volume percentage receiving $105 \%$ or more of the prescribed dose). Dose distribution in the axillary lymph nodes was evaluated using the mean axillary lymph nodes levels I, II and III doses. The OAR constraints used for planning for the ipsilateral lung were the mean ipsilateral lung dose (MLD), ipV20 (the lung volume percentage receiving $\geq 20 \mathrm{~Gy}$ ) and ipV30 (the lung volume percentage receiving $\geq 30 \mathrm{~Gy}$ ), while those for the heart were the mean heart dose (MHD), V10 (the heart volume percentage receiving $\geq 10 \mathrm{~Gy}$ ), V20 (the heart volume percentage receiving $\geq 20 \mathrm{~Gy}$ ), and the maximum doses to the left anterior descending coronary artery (LAD), the circumflex coronary artery (CCA) and the right coronary artery (RCA). 
Acute toxicity grading for skin. Patients were evaluated on a weekly basis for acute skin toxicities. The highest grade of radiation dermatitis for each treated breast was prospectively recorded at each on treatment during the 5 or 6 weeks of treatment and at the follow-up visit at the week 6 or 7 for patients who received RT without or with boost, respectively, using the National Cancer Institute Common Terminology Criteria for Adverse Events (NCI CTCAE) v3.0 grading criteria (12). The NCI CTCAE describes grade 0 as 'no reaction', grade 1 as 'faint erythema or dry desquamation', grade 2 as 'moderate to brisk erythema, patchy moist desquamation mostly confined to skin folds and creases and moderate edema', grade 3 as 'moist desquamation other than skin folds or creases and bleeding induced by minor trauma or abrasion' and grade 4 as 'skin necrosis or ulceration of full thickness of the dermis, spontaneous bleeding from involved site' $(3,12)$. To ensure standard grading of skin toxicities for study purposes, all information regarding toxicity grade in individual patient were recorded and reviewed by a single experienced radiation oncologist.

Data collection and statistical analysis. Demographic characteristics and clinical tumor characteristics were recorded for each patient. Tumor site was defined according to the International Classification of Diseases for Oncology (third edition) (13). Each radiation treatment plan was assessed, and dosimetric parameters were analyzed using a dose-volume histogram. For this study, acute toxicity grading for skin within the first 5 weeks of treatment was analyzed.

Statistical analyses were performed using Bell Curve for Excel (Social Survey Research Information Co., Ltd.) and SPSS software v27.0 (IBM Corp.). Analyses of differences in the baseline characteristics, the RT characteristics, the skin toxicity grade throughout the RT treatment and the maximum skin toxicity grade during the 5 weeks of RT treatment according to the type of irradiation method used were conducted using the Kruskal-Wallis test with Dunn's test and the $\chi^{2}$ test. The correlation between the irradiation methods and the dose-distribution factors was examined to assess the impact of irradiation methods on the dose-distribution factors, using Eta correlation ratio $(\eta)$. The correlation between the skin toxicity grade and patients' baseline characteristics was analyzed using $\eta$ and Spearman's correlation coefficient (rs), and the correlation between the skin toxicity grade and irradiation methods was analyzed using $\eta$. The following proposed guidelines were used for interpreting the absolute values of $\eta$ and rs: 0.00-0.19, markedly weak; 0.20-0.39, weak; 0.40-0.59, moderate; 0.60-0.79, strong; and 0.80-1.00, markedly strong. Data are presented as the mean $\pm \mathrm{SD}, \mathrm{n}(\%)$, $\eta$ or rs values. $\mathrm{P}<0.05$ was considered to indicate a statistically significant difference.

\section{Results}

Baseline characteristics according to the irradiation methods are detailed in Table I. Age, tumor site, breast volume or separation did not show statistically significant differences between the three different irradiation methods (Table I).

Dosimetric analysis. RT characteristics according to the irradiation methods are detailed in Table II. PTV volume did not show a statistically significant difference between the three different irradiation methods. However, a significant impact of the irradiation method was observed on PTV and BPe dosimetric parameters, the mean dose, V0-95\%, V95-107\%, $\mathrm{V} 107 \%$ and $\mathrm{V} 105 \%$. The volumes of the PTV and BPe receiving $\geq 105 \%$ of the prescribed dose were the lowest when using the FIF w/ 2RP method, with mean \pm standard deviation (SD) values of $0.09 \pm 0.75$ and $0.10 \pm 0.90$, respectively. The mean axillary lymph nodes levels I, II and III doses were intermediate when using the FIF w/ 2RP method, with mean \pm SD values of $3,278 \pm 822,1,455 \pm 1,127$ and $427 \pm 528$, respectively. OAR constraints showed statistically significant differences between three different irradiation methods. The MHD, and the maximum doses to the CCA and the RCA were the lowest when using the FIF w/ 2RP method, with mean \pm SD values of $248 \pm 76,201 \pm 32$ and $212 \pm 34$, respectively. However, the heart $\mathrm{V} 10$ and V20, and the maximum doses to the LAD were intermediate when using the FIF w/ 2RP method, with mean \pm SD values of $3.0 \pm 2.1,1.6 \pm 1.5$ and $3,123 \pm 1,334$, respectively. Nevertheless, the MLD, ipV20 and ipV30 were the highest when using the FIF w/ 2RP method, with mean \pm SD values of $771 \pm 198,14 \pm 5$ and $11 \pm 4$, respectively (Table II).

Table III shows that the irradiation methods strongly affected the dose distribution factors. There were strong associations between all irradiation methods and the volumes of the PTV and BPe receiving $\geq 105 \%$ of the prescribed dose with $(\eta=0.62, P<0.001)$ and $(\eta=0.61, P<0.001)$, respectively. The strongest associations were found when comparing the FIF w/ 2RP method vs. the FIF w/o 2RP method for PTV V105\% $(\eta=0.79, P<0.001)$ and BPe V105\% $(\eta=0.76, P<0.001)$. Other dose distribution factors were less affected by the irradiation methods, with markedly weak to moderate $\eta$ values. For the heart, all irradiation methods had a weak association with MHD $(\eta=0.26, P<0.001)$, and moderate associations with maximum doses to the CCA $(\eta=0.45, \mathrm{P}<0.001)$ and to the RCA $(\eta=0.43, P<0.001)$. The strongest associations were found when comparing the FIF w/ 2RP method vs. the W method for MHD $(\eta=0.29, P<0.001)$, maximum doses to the CCA $(\eta=0.50, P<0.001)$ and to the RCA $(\eta=0.47, P<0.001)$. For the lung, all irradiation methods had weak associations with $\operatorname{MLD}(\eta=0.28, P<0.001)$, ipV20 $(\eta=0.31, P<0.001)$ and ipV30 $(\eta=0.34, P<0.001)$. The strongest associations were found when comparing the FIF w/ 2RP method vs. the W method for MLD $(\eta=0.28, P<0.001)$, ipV20 $(\eta=0.30, P<0.001)$ and ipV30 $(\eta=0.33, P<0.001)$.

Skin toxicity analysis. All irradiation methods were well tolerated, since no case of treatment interruption due to acute skin toxicity neither grade 3 skin toxicities were documented (Figs. 1 and 2).

The association between the time course of the RT and the mean skin toxicity grade registered at weeks $1-5$, and the maximum skin toxicity grade during the 5 weeks of RT treatment for each irradiation method are shown in Fig. 1. The occurrence and mean skin toxicity grade increased from the start of the RT for all the irradiation methods, especially after 3 weeks of RT. In weeks 3 and 4, the FIF w/ 2RP method had a significant impact on lowering the skin toxicity mean grade. Kruskal-Wallis test showed that there were significant differences in the skin toxicity median grade during weeks 2 to 5 (Fig. 1). 
Table I. Baseline characteristics according to the irradiation methods.

A, All patients

\begin{tabular}{|c|c|c|c|c|c|}
\hline \multirow[b]{2}{*}{ Variable } & \multirow[b]{2}{*}{ All irradiation methods } & \multicolumn{3}{|c|}{ Irradiation method } & \multirow[b]{2}{*}{ P-value } \\
\hline & & $\mathrm{W}$ & FIF w/o 2RP & FIF w/ 2RP & \\
\hline Patients, $\mathrm{n}$ & 573 & 178 & 142 & 253 & \\
\hline Mean age $\pm \mathrm{SD}$, years & $55 \pm 11$ & $54 \pm 11$ & $55 \pm 12$ & $55 \pm 11$ & $\mathrm{P}=0.38^{\mathrm{a}}$ \\
\hline Tumor site, n (\%) & & & & & $\mathrm{P}=0.29^{\mathrm{b}}$ \\
\hline Upper-inner quadrant & $161(28.1)$ & $47(26.4)$ & $48(33.8)$ & $66(26.1)$ & \\
\hline Lower-inner quadrant & $50(8.7)$ & $18(10.1)$ & $8(5.6)$ & $24(9.5)$ & \\
\hline Upper-outer quadrant & $278(48.5)$ & $81(45.5)$ & $64(45.1)$ & $133(52.6)$ & \\
\hline Lower-outer quadrant & $55(9.6)$ & $23(12.9)$ & $14(9.9)$ & $18(7.1)$ & \\
\hline Central portion & $29(5.1)$ & $9(5.1)$ & $8(5.6)$ & $12(4.7)$ & \\
\hline Mean $\mathrm{BPe} \pm \mathrm{SD}, \mathrm{cm}^{3}$ & $442 \pm 254$ & $457 \pm 271$ & $428 \pm 244$ & $439 \pm 247$ & $\mathrm{P}=0.77^{\mathrm{a}}$ \\
\hline Mean separation $\pm \mathrm{SD}, \mathrm{cm}$ & $19.1 \pm 2.4$ & $19.1 \pm 2.3$ & $18.7 \pm 2.4$ & $19.3 \pm 2.5$ & $\mathrm{P}=0.07^{\mathrm{a}}$ \\
\hline
\end{tabular}

B, Left-sided patients

\begin{tabular}{|c|c|c|c|c|c|}
\hline \multirow[b]{2}{*}{ Variable } & \multirow[b]{2}{*}{ All irradiation methods } & \multicolumn{3}{|c|}{ Irradiation method } & \multirow[b]{2}{*}{ P-value } \\
\hline & & $\mathrm{W}$ & FIF w/o 2RP & FIF w/ 2RP & \\
\hline Patients, $\mathrm{n}$ & 296 & 89 & 70 & 137 & \\
\hline Mean age $\pm S D$, years & $55 \pm 11$ & $55 \pm 11$ & $57 \pm 11$ & $55 \pm 11$ & $\mathrm{P}=0.26^{\mathrm{a}}$ \\
\hline Tumor site, $\mathrm{n}(\%)$ & & & & & $\mathrm{P}=0.75^{\mathrm{b}}$ \\
\hline Upper-inner quadrant & $82(27.7)$ & $26(29.2)$ & $23(32.9)$ & $33(24.1)$ & \\
\hline Lower-inner quadrant & $30(10.1)$ & $11(12.4)$ & $4(5.7)$ & $15(10.9)$ & \\
\hline Upper-outer quadrant & $149(50.3)$ & $42(47.2)$ & $34(48.6)$ & $73(53.3)$ & \\
\hline Lower-outer quadrant & $16(5.4)$ & $6(6.7)$ & $4(5.7)$ & $6(4.4)$ & \\
\hline Central portion & $19(6.4)$ & $4(4.5)$ & $5(7.1)$ & $10(7.3)$ & \\
\hline Mean $\mathrm{BPe} \pm \mathrm{SD}, \mathrm{cm}^{3}$ & $442 \pm 253$ & $471 \pm 255$ & $430 \pm 260$ & $429 \pm 249$ & $\mathrm{P}=0.38^{\mathrm{a}}$ \\
\hline Mean separation $\pm \mathrm{SD}, \mathrm{cm}$ & $18.9 \pm 2.6$ & $19.1 \pm 2.5$ & $18.6 \pm 2.7$ & $18.9 \pm 2.5$ & $\mathrm{P}=0.37^{\mathrm{a}}$ \\
\hline
\end{tabular}

The percentage of patients according to the maximum skin toxicity grade during the 5 weeks of RT treatment for each irradiation method is summarized in Fig. 2. For the three irradiation methods, the maximum skin toxicity grade recorded was grade 2 . The irradiation methods were significantly associated with the appearance of adverse effects. The use of the FIF w/ 2RP method increased the occurrence of grade 0 skin toxicity. Grade 0 was observed in $2(1.1 \%), 0$ $(0.0 \%)$ and $14(5.5 \%)$ cases, respectively, for the W, FIF w/o 2RP and FIF w/ 2RP methods. Grade 0 for the FIF w/ 2RP method was significantly higher compared to the other irradiation methods. Furthermore, the use of the FIF w/ 2RP method decreased the occurrence of grade 1 skin toxicity. Grade 1 was observed in 168 (94.4\%), 139 (97.9\%) and 231 (91.3\%) cases, respectively, for the W, FIF w/o 2RP and FIF w/ 2RP methods. However, grade 2 skin toxicity had an intermediate percentage when using the FIF w/ 2RP method. Grade 2 was observed in $8(4.5 \%), 3(2.1 \%)$ and $8(3.2 \%)$ cases, respectively, for the W, FIF w/o 2RP and FIF w/ 2RP methods.

For associations related to skin toxicity grade, results were examined at the following three time points: Week 3, week 4 and maximum skin toxicity grade during the 5 weeks of RT treatment. As shown in Fig. 1, the skin toxicity grades from the start of the RT treatment to the 2nd week were low; moreover, the skin toxicity grade at week 5 and the maximum grade during the 5 weeks of RT treatment were similar. Therefore, associations of skin toxicity grade were examined at these three time points.

The association between patients' baseline characteristics and skin toxicity grade was analyzed for all patients. Most significant associations were observed in the following baseline characteristics: Breast volume factors (BPe volume, separation 
Table II. Radiotherapy characteristics according to the irradiation methods.

A, All patients

Irradiation method

\begin{tabular}{|c|c|c|c|c|}
\hline \multirow[b]{2}{*}{ Variable } & \multicolumn{3}{|c|}{ Irradiation method } & \multirow[b]{2}{*}{ P-value } \\
\hline & $\mathrm{W}$ & FIF w/o 2RP & FIF w/ 2RP & \\
\hline Patients, $\mathrm{n}$ & 178 & 142 & 253 & \\
\hline \multicolumn{5}{|l|}{ Target } \\
\hline \multicolumn{5}{|l|}{ PTV } \\
\hline Volume, $\mathrm{cm}^{3}$ & $592 \pm 324$ & $573 \pm 303$ & $571 \pm 304$ & $\mathrm{P}=0.93$ \\
\hline Mean dose, cGy & $4,924 \pm 84$ & $5,011 \pm 65$ & $4,952 \pm 45$ & $\mathrm{P}<0.001\left(\mathrm{P}<0.01^{\mathrm{c}}, \mathrm{P}<0.001^{\mathrm{b}, \mathrm{d}}\right)$ \\
\hline V0-95, \% & $15 \pm 8$ & $10 \pm 7$ & $13 \pm 7$ & $\mathrm{P}<0.001\left(\mathrm{P}<0.05^{\mathrm{c}}, \mathrm{P}<0.001^{\mathrm{b}, \mathrm{d}}\right)$ \\
\hline V95-107, \% & $84 \pm 8$ & $90 \pm 7$ & $87 \pm 7$ & $\mathrm{P}<0.001\left(\mathrm{P}<0.01^{\mathrm{c}}, \mathrm{P}<0.001^{\mathrm{b}, \mathrm{d}}\right)$ \\
\hline V107, \% & $0.52 \pm 1.50$ & $0.27 \pm 0.28$ & $0.00 \pm 0.01$ & $\mathrm{P}<0.001\left(\mathrm{P}<0.001^{\mathrm{b}-\mathrm{d}}\right)$ \\
\hline $\mathrm{V} 105, \%$ & $3.50 \pm 4.88$ & $6.63 \pm 3.96$ & $0.09 \pm 0.75$ & $\mathrm{P}<0.001\left(\mathrm{P}<0.001^{\mathrm{b}-\mathrm{d}}\right)$ \\
\hline \multicolumn{5}{|l|}{ Breast PTV evaluation } \\
\hline Mean dose, cGy & $4,969 \pm 62$ & $5,043 \pm 60$ & $4,980 \pm 48$ & $\mathrm{P}<0.001\left(\mathrm{P}<0.001^{\mathrm{b}, \mathrm{d}}\right)$ \\
\hline $\mathrm{V} 0-95, \%$ & $10 \pm 6$ & $5 \pm 5$ & $8 \pm 7$ & $\mathrm{P}<0.001\left(\mathrm{P}<0.01^{\mathrm{c}}, \mathrm{P}<0.001^{\mathrm{b}, \mathrm{d}}\right)$ \\
\hline V95-107, \% & $90 \pm 6$ & $95 \pm 5$ & $92 \pm 7$ & $\mathrm{P}<0.001\left(\mathrm{P}<0.001^{\mathrm{b}-\mathrm{d}}\right)$ \\
\hline V107, \% & $0.42 \pm 1.45$ & $0.25 \pm 0.35$ & $0.00 \pm 0.01$ & $\mathrm{P}<0.001\left(\mathrm{P}<0.001^{\mathrm{b}-\mathrm{d}}\right)$ \\
\hline $\mathrm{V} 105, \%$ & $3.24 \pm 4.86$ & $6.92 \pm 4.56$ & $0.10 \pm 0.90$ & $\mathrm{P}<0.001\left(\mathrm{P}<0.001^{\mathrm{b}-\mathrm{d}}\right)$ \\
\hline \multicolumn{5}{|l|}{ Axillary lymph node } \\
\hline \multicolumn{5}{|l|}{ Mean dose, cGy } \\
\hline Level I & $2,928 \pm 717$ & $3,529 \pm 750$ & $3,278 \pm 822$ & $\mathrm{P}<0.001\left(\mathrm{P}<0.05^{\mathrm{d}}, \mathrm{P}<0.001^{\mathrm{b}, \mathrm{c}}\right)$ \\
\hline Level II & $1,356 \pm 1,029$ & $1,726 \pm 1,330$ & $1,455 \pm 1,127$ & $\mathrm{P}=0.10$ \\
\hline Level III & $357 \pm 399$ & $508 \pm 566$ & $427 \pm 528$ & $\mathrm{P}<0.05\left(\mathrm{P}<0.05^{\mathrm{b}}\right)$ \\
\hline \multicolumn{5}{|l|}{ Organ at risk: Lung } \\
\hline ip mean dose (cGy) & $659 \pm 177$ & $655 \pm 200$ & $771 \pm 198$ & $\mathrm{P}<0.001\left(\mathrm{P}<0.001^{\mathrm{c}, \mathrm{d}}\right)$ \\
\hline ipV20 (\%) & $11 \pm 4$ & $11 \pm 5$ & $14 \pm 5$ & $\mathrm{P}<0.001\left(\mathrm{P}<0.001^{\mathrm{c}, \mathrm{d}}\right)$ \\
\hline ipV30 (\%) & $8 \pm 4$ & $8 \pm 4$ & $11 \pm 4$ & $\mathrm{P}<0.001\left(\mathrm{P}<0.001^{\mathrm{c}, \mathrm{d}}\right)$ \\
\hline
\end{tabular}

B, Left-sided patients

Irradiation method

\begin{tabular}{|c|c|c|c|c|}
\hline \multirow[b]{2}{*}{ Variable } & & & & \multirow[b]{2}{*}{ P-value } \\
\hline & $\mathrm{W}$ & FIF w/o 2RP & FIF w/ 2RP & \\
\hline Patients, $\mathrm{n}$ & 89 & 70 & 137 & \\
\hline \multicolumn{5}{|l|}{ Target } \\
\hline \multicolumn{5}{|l|}{ PTV } \\
\hline Volume, $\mathrm{cm}^{3}$ & $610 \pm 322$ & $588 \pm 325$ & $555 \pm 300$ & $\mathrm{P}=0.45$ \\
\hline \multicolumn{5}{|l|}{ Organ at risk: Heart } \\
\hline Mean dose, $\mathrm{cGy}$ & $300 \pm 99$ & $257 \pm 90$ & $248 \pm 76$ & $\mathrm{P}<0.001\left(\mathrm{P}<0.01^{\mathrm{b}}, \mathrm{P}<0.001^{\mathrm{c}}\right)$ \\
\hline $\mathrm{V} 10, \%$ & $3.8 \pm 2.9$ & $2.9 \pm 2.6$ & $3.0 \pm 2.1$ & $\mathrm{P}<0.05\left(\mathrm{P}<0.05^{\mathrm{b}}\right)$ \\
\hline $\mathrm{V} 20, \%$ & $2.0 \pm 2.1$ & $1.4 \pm 1.8$ & $1.6 \pm 1.5$ & $\mathrm{P}=0.08$ \\
\hline LAD max, cGy & $3,546 \pm 1,228$ & $2,931 \pm 1,490$ & $3,123 \pm 1,334$ & $\mathrm{P}<0.05\left(\mathrm{P}<0.05^{\mathrm{b}, \mathrm{c}}\right)$ \\
\hline CCA max, cGy & $239 \pm 32$ & $212 \pm 31$ & $201 \pm 32$ & $\mathrm{P}<0.001\left(\mathrm{P}<0.001^{\mathrm{b}, \mathrm{c}}\right)$ \\
\hline RCA max, cGy & $262 \pm 59$ & $228 \pm 39$ & $212 \pm 34$ & $\mathrm{P}<0.001\left(\mathrm{P}<0.01^{\mathrm{d}}, \mathrm{P}<0.001^{\mathrm{b}, \mathrm{c}}\right)$ \\
\hline
\end{tabular}

The data are presented as the mean $\pm \mathrm{SD}$. Left-sided patients refers to patients who received left-sided breast radiotherapy. ${ }^{\mathrm{a}} \mathrm{W}$ vs. FIF w/o $2 \mathrm{RP}$ vs. FIF w/ 2RP via Kruskal-Wallis test; ${ }^{b} \mathrm{~W}$ vs. FIF w/o 2RP; ${ }^{c} \mathrm{~W}$ vs. FIF w/ 2RP; and ${ }^{\mathrm{d}}$ FIF w/o 2RP vs. FIF w/ 2RP via Dunn's test. W, wedge; FIF w/o 2RP, field-in-field without 2 reference points; FIF w/ 2RP, field-in-field with 2 reference points; PTV, planning target volume; ip, ipsilateral; LAD, left anterior descending artery; CCA, common carotid artery; RCA, right coronary artery; max, maximum dose. 
Table III. Association between irradiation methods and dose-distribution factors.

\begin{tabular}{|c|c|c|c|c|}
\hline Variable & $\begin{array}{l}\text { All irradiation } \\
\text { methods }\end{array}$ & $\begin{array}{l}\text { W vs. FIF } \\
\text { w/o } 2 \text { RP }\end{array}$ & $\begin{array}{l}\text { W vs. FIF } \\
\text { w/ } 2 \text { RP }\end{array}$ & $\begin{array}{c}\text { FIF w/o 2RP vs. } \\
\text { FIF w/ 2RP }\end{array}$ \\
\hline \multicolumn{5}{|l|}{ Target } \\
\hline \multicolumn{5}{|l|}{ High dose region, $\%$} \\
\hline PTV V107 & $0.26^{\mathrm{a}}$ & $0.11^{\mathrm{b}}$ & $0.26^{\mathrm{a}}$ & $0.61^{\mathrm{a}}$ \\
\hline PTV V105 & $0.62^{\mathrm{a}}$ & $0.33^{\mathrm{a}}$ & $0.47^{\mathrm{a}}$ & $0.79^{a}$ \\
\hline BPe V107 & $0.21^{\mathrm{a}}$ & $0.08(\mathrm{P}=0.17)$ & $0.21^{\mathrm{a}}$ & $0.49^{\mathrm{a}}$ \\
\hline BPe V105 & $0.61^{\mathrm{a}}$ & $0.36^{\mathrm{a}}$ & $0.44^{\mathrm{a}}$ & $0.76^{\mathrm{a}}$ \\
\hline \multicolumn{5}{|c|}{ Adequate dose region, $\%$} \\
\hline PTV V95-107 & $0.28^{\mathrm{a}}$ & $0.36^{\mathrm{a}}$ & $0.17^{\mathrm{a}}$ & $0.21^{\mathrm{a}}$ \\
\hline BPe V95-107 & $0.28^{\mathrm{a}}$ & $0.40^{\mathrm{a}}$ & $0.14^{\mathrm{a}}$ & $0.23^{\mathrm{a}}$ \\
\hline \multicolumn{5}{|l|}{ Low dose region, $\%$} \\
\hline PTV V0-95 & $0.26^{\mathrm{a}}$ & $0.33^{\mathrm{a}}$ & $0.13^{\mathrm{b}}$ & $0.22^{\mathrm{a}}$ \\
\hline BPe V0-95 & $0.27^{\mathrm{a}}$ & $0.38^{\mathrm{a}}$ & $0.11^{\mathrm{b}}$ & $0.24^{\mathrm{a}}$ \\
\hline \multicolumn{5}{|l|}{ Mean dose, cGy } \\
\hline PTV & $0.45^{\mathrm{a}}$ & $0.49^{\mathrm{a}}$ & $0.21^{\mathrm{a}}$ & $0.47^{\mathrm{a}}$ \\
\hline $\mathrm{BPe}$ & $0.47^{\mathrm{a}}$ & $0.52^{\mathrm{a}}$ & $0.10^{\mathrm{b}}$ & $0.50^{\mathrm{a}}$ \\
\hline \multicolumn{5}{|c|}{ Axillary lymph node mean dose, cGy } \\
\hline Level I & $0.28^{\mathrm{a}}$ & $0.38^{\mathrm{a}}$ & $0.22^{\mathrm{a}}$ & $0.15^{\mathrm{b}}$ \\
\hline Level II & $0.12^{\mathrm{b}}$ & $0.16^{\mathrm{b}}$ & $0.05(\mathrm{P}=0.35)$ & $0.11^{\mathrm{b}}$ \\
\hline Level III & $0.11^{\mathrm{b}}$ & $0.15^{\mathrm{b}}$ & $0.07(\mathrm{P}=0.14)$ & $0.07(\mathrm{P}=0.16)$ \\
\hline \multicolumn{5}{|l|}{ Organs at risk } \\
\hline \multicolumn{5}{|l|}{ Lung } \\
\hline ip mean dose, cGy & $0.28^{\mathrm{a}}$ & $0.01(\mathrm{P}=0.84)$ & $0.28^{\mathrm{a}}$ & $0.27^{\mathrm{a}}$ \\
\hline ipV20,\% & $0.31^{\mathrm{a}}$ & $0.02(\mathrm{P}=0.75)$ & $0.30^{\mathrm{a}}$ & $0.30^{\mathrm{a}}$ \\
\hline $\mathrm{ipV} 30, \%$ & $0.34^{\mathrm{a}}$ & $0.00(\mathrm{P}=0.94)$ & $0.33^{\mathrm{a}}$ & $0.31^{\mathrm{a}}$ \\
\hline \multicolumn{5}{|c|}{ Heart (left-sided patients) } \\
\hline Mean dose, cGy & $0.26^{\mathrm{a}}$ & $0.22^{\mathrm{b}}$ & $0.29^{\mathrm{a}}$ & $0.05(\mathrm{P}=0.45)$ \\
\hline $\mathrm{V} 10, \%$ & $0.16^{\mathrm{b}}$ & $0.17^{\mathrm{b}}$ & $0.16^{\mathrm{b}}$ & $0.03(\mathrm{P}=0.68)$ \\
\hline $\mathrm{V} 20, \%$ & $0.13(\mathrm{P}=0.07)$ & $0.15(\mathrm{P}=0.06)$ & $0.12(\mathrm{P}=0.06)$ & $0.05(\mathrm{P}=0.50)$ \\
\hline LAD max, cGy & $0.17^{\mathrm{b}}$ & $0.22^{\mathrm{b}}$ & $0.16^{\mathrm{b}}$ & $0.07(\mathrm{P}=0.35)$ \\
\hline CCA max, cGy & $0.45^{\mathrm{a}}$ & $0.39^{\mathrm{a}}$ & $0.50^{\mathrm{a}}$ & $0.17^{\mathrm{b}}$ \\
\hline RCA max, cGy & $0.43^{\mathrm{a}}$ & $0.31^{\mathrm{a}}$ & $0.47^{\mathrm{a}}$ & $0.20^{\mathrm{b}}$ \\
\hline
\end{tabular}

The data are presented as the Eta correlation ratio $(\eta)$ values. Left-sided patients refers to patients who received left-sided breast radiotherapy. ${ }^{a} \mathrm{P}<0.001$ and ${ }^{\mathrm{b}} \mathrm{P}<0.05$ via Eta correlation ratio test. W, wedge; FIF w/o $2 \mathrm{RP}$, field-in-field without 2 reference points; FIF w/ 2RP, field-in-field with 2 reference points; PTV, planning target volume; BPe, breast PTV evaluation; ip, ipsilateral; LAD, left anterior descending artery; $\mathrm{CCA}$, common carotid artery; RCA, right coronary artery; max, maximum dose.

and PTV volume) and weight exhibited markedly weak positive correlations with the maximum skin toxicity grade during the 5 weeks of RT treatment. The rs values were as follows for BPe volume ( $r s=0.17, \mathrm{P}<0.01)$, separation ( $\mathrm{rs}=0.17, \mathrm{P}<0.01$ ), PTV volume $(\mathrm{rs}=0.18, \mathrm{P}<0.01)$ and weight $(\mathrm{rs}=0.17, \mathrm{P}<0.01)$. Tumor site exhibited markedly weak associations with the skin toxicity grade at weeks 3 and 4 . The $\eta$ values were as follows for tumor site at week $3(\eta=0.16, \mathrm{P}<0.05)$ and at week 4 $(\eta=0.16, P<0.05)$.

Table IV shows the association between irradiation methods and skin toxicity grade. The impact of the irradiation methods differed with the time course of the RT treatment. Associations between all irradiation methods and the skin toxicity grade were moderate at week $3(\eta=0.46, P<0.001)$, weak at week $4(\eta=0.33, P<0.001)$ and markedly weak for the maximum skin toxicity grade during the 5 weeks of RT treatment $(\eta=0.11, P<0.05)$. Significant associations were found at all time points in the FIF w/ 2RP method vs. the W method group, but only at weeks 3 and 4 in the FIF w/ 2RP method vs. the FIF w/o 2RP method group.

\section{Discussion}

In accordance with the modern RT goal of reducing skin toxicity and improving the quality of life of patients with early-stage breast cancer, our study aimed to present an irradiation method that is both clinically practical and effective in achieving lower skin toxicity rates. This study showed that the 


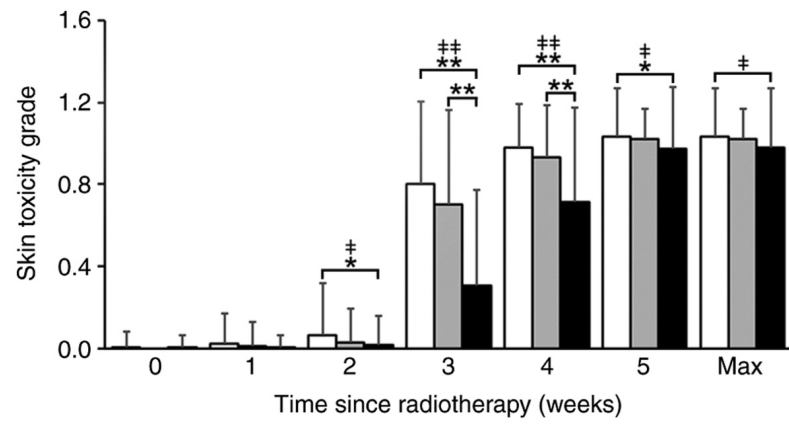

Figure 1. Association between the time course of radiotherapy and skin toxicity grade. ${ }^{\dagger} \mathrm{P}<0.05$ and ${ }^{\sharp} \mathrm{P}<0.001$ via Kruskal-Wallis test; ${ }^{*} \mathrm{P}<0.05$ and ${ }^{* *} \mathrm{P}<0.001$ via Dunn's test. W, wedge; FIF w/o 2RP, field-in-field without 2 reference points; FIF w/ 2RP, field-in-field with 2 reference points; Max, maximum skin toxicity grade during the 5 weeks of radiotherapy treatment.

FIF w/ 2RP method, compared with the W and FIF w/o 2RP methods, was advantageous in maintaining a homogeneous dose distribution while reducing high-dose areas in the target and doses at OARs, and consequently lowering skin toxicity occurrence and grades.

For WBRT, several RT techniques are available to achieve a uniform and standardized dose throughout the whole breast. The progression of RT techniques and delivery systems, including intensity-modulated radiation therapy (IMRT), volumetric modulated arc therapy (VMAT) and deep inspiration breath-hold methods, improved dose homogeneity and enabled a better dose distribution. However, the 3D-CRT treatment planning is a widespread implemented system, and when used in adjunction to the FIF technique, enables an improved homogeneity in the irradiated volume and lowers high-dose areas $(9,14)$.

Wright et al (15) evaluated 392 patients, $83 \%$ of which were treated with conventionally fractionated scheme and $17 \%$ with a hypofractionated scheme. Regional nodal radiation was delivered in $15 \%$ of patients, and the dose range was 45.0-50.4 Gy in 25 fractions. A boost to the lumpectomy cavity of 10-16 Gy was received by $88 \%$ of patients. These patients had $35 \%$ of V105\% and $16 \%$ of V110\% (15). Giri et al (16) compared three techniques (VMAT, FIF and W methods) in 20 patients, and indicated that VMAT is a superior method compared with the other two methods. The authors reported that V105\% for PTV using VMAT was 3.3 $\pm 5.5 \%$. Our V105\% using the FIF w/ 2RP method was better than this value. Osei et al (17) conducted a dose-volumetric analysis for the normalized PTV_eval for patients treated with hybrid inverse planned IMRT technique (50-Gy dose in 25 fractions). The mean PTV_eval V105\% was $0.81 \%$ for all patients with a mean breast separation of $22.21 \mathrm{~cm}$, whereas the mean PTV_eval V105\% was $0.37 \%$ for patients with a mean breast separation $<20 \mathrm{~cm}$. Compared with these results and with the use of the FIF w/o 2RP method, the use of the FIF w/ 2RP method resulted in values of PTV $\mathrm{V} 105 \%$ as low as $0.09 \%$.

The impact of the irradiation method used on dose distribution to target volumes was further elucidated by the significantly strong associations in high-dose regions, particularly in the FIF w/o 2RP method vs. FIF w/ 2RP method group, confirming that our method significantly decreases high-dose region volumes.

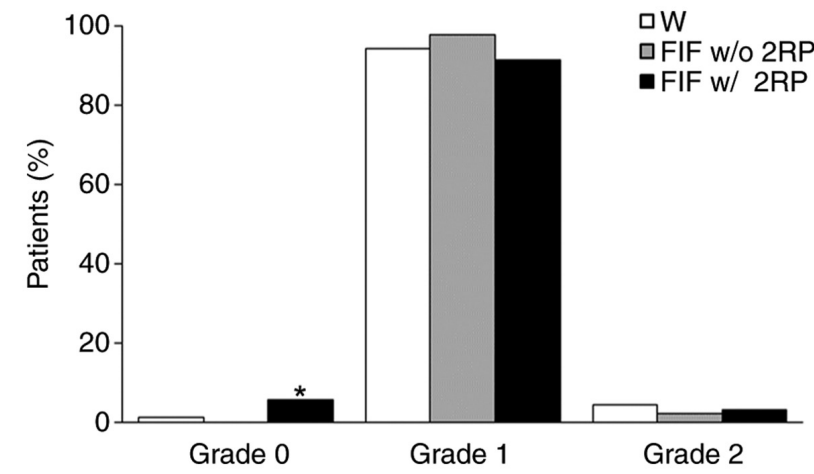

Figure 2. Percentage of patients for each skin toxicity grade according to the irradiation methods. The presented skin toxicity grades are the maximum skin toxicity grades that occurred during the 5 weeks of radiotherapy treatment. ${ }^{*} \mathrm{P}<0.006$ via $\chi^{2}$ test with Bonferroni correction was considered to indicate a statically significant difference. W, wedge; FIF w/o 2RP, field-in-field without 2 reference points; FIF w/ 2RP, field-in-field with 2 reference points.

As for OARs, particularly dose distribution in the heart, Duma et al (18) assessed the heart dosimetry parameters in patients with breast cancer using 3D-CRT by tangential fields, half beam technique. For a group of patients with an estimated low dose to the heart, the authors reported a median MHD to the whole heart of $2.6 \mathrm{~Gy}(0.8-3.5 \mathrm{~Gy}), \mathrm{V} 10$ of $3.4 \%$ and V20 of $2.5 \%$ (18). The values reported in our study are lower, thus improving dose distribution in the heart and lowering the risk of late cardiac radiation injuries. Beaton et al (19) identified all cardiovascular deaths in women with early-stage breast cancer treated with breast/chest wall RT. The authors found that the radiation-induced cardiac death at 10 years was low if MHD is <3.3 Gy, maximum LAD dose is <45.4 Gy and V25 $<5 \%$ (19). For each of the three used irradiation methods, our study calculated the percentage of patients receiving MHD <3.3 Gy and maximum LAD dose <45.4 Gy. It was found that the FIF w/ 2RP method had higher percentage of cases with MHD <3.3 Gy $(86.1 \%)$ and higher percentage of cases with maximum LAD dose $<45.4$ Gy $(86.9 \%)$ compared with those subjected to the W and FIF w/o 2RP methods, thus suggesting that FIF w/ 2RP might be a better method to reduce radiation-induced cardiac death at 10 years.

For dose distribution in the lung, Chung et al (20) reported that the dosimetric parameter MLD using the partially wide tangent technique is correlated with the incidence of radiation pneumonitis. There was a statistically significant difference in the incidence of radiation pneumonitis between patients who had an MLD of 17.9 \pm 3.2 and patients who had an MLD of 19.3 \pm 2.8 . The MLD was significantly different between total radiation pneumonitis grade 0 and grade $\geq 1(\mathrm{P}=0.042)$. MLD of 20.5 Gy was determined as the cut-off point for the incidence of radiation pneumonitis. However, the average value of V20 and V30 showed no statistically significant difference in the incidence of radiation pneumonitis. Patients with an average value of $\mathrm{V} 20$ of $36.1 \pm 7.7 \%$ and $\mathrm{V} 30$ of $31.3 \pm 7.1 \%$ had no radiation pneumonitis (20). Although the FIF w/ 2RP method had higher values than the FIF w/o 2RP method, these values are still considerably low compared with the values reported to have a correlation with the incidence of radiation pneumonitis. Therefore, the difference in lung doses within the used irradiation methods may not have clinical 
Table IV. Association between irradiation methods and skin toxicity grade.

\begin{tabular}{|c|c|c|c|c|}
\hline Variable & All irradiation methods & W vs. FIF w/o 2RP & W vs. FIF w/ 2RP & FIF w/o 2RP vs. FIF w/ 2RP \\
\hline \multicolumn{5}{|l|}{ Skin toxicity grade } \\
\hline Week 3 & $0.46^{\mathrm{a}}$ & $0.12^{\mathrm{b}}$ & $0.49^{\mathrm{a}}$ & $0.38^{\mathrm{a}}$ \\
\hline Week 4 & $0.33^{\mathrm{a}}$ & $0.10(\mathrm{P}=0.07)$ & $0.33^{\mathrm{a}}$ & $0.25^{\mathrm{a}}$ \\
\hline Max week (0-5) & $0.11^{\mathrm{b}}$ & $0.03(\mathrm{P}=0.58)$ & $0.10^{\mathrm{b}}$ & $0.09(\mathrm{P}=0.09)$ \\
\hline
\end{tabular}

significance. Yang et al (21) compared wedge and FIF IMRT techniques in patients with small-sized breasts. The authors suggested that the conventional FIF method has favorable dose conformity and is an optimal method in patients with breast volume $\leq 350 \mathrm{~cm}^{3}$. Using the conventional FIF method, the authors reported a lung V20\% of $14.42 \pm 2.61 \%$ and a heart $\mathrm{V} 25 \%$ of $2.44 \pm 1.07 \%$ (21). Although our patients had a mean breast volume of $493 \pm 247$ cc using the FIF w/ 2RP method, our results indicated a similar value for the lung V20\% of $14 \pm 5 \%$ and a lower value for the heart V20\% of $1.6 \pm 1.5 \%$. Morganti et al (22), using a forward FIF IMRT technique for 201 patients with a mean breast volume of $528 \mathrm{cc}$, reported that $\mathrm{V} 107 \%$ of the irradiated volume was $2.4 \%$, which is higher than the $0 \%$ value observed in our patients treated with the FIF w/ 2RP method. The authors also reported MLD and MHD values of 9.1 and $3.4 \mathrm{~Gy}$, respectively, whereas our FIF w/ 2RP method could reduce the MLD and MHD to 7.7 and 2.5 Gy, respectively.

The existence of a high-dose range in the irradiation field and its association to skin toxicities is reported in the literature (6-8). Chen et al (7) reported that, for patients treated with $\mathrm{BCS}$, higher volume receiving PTV-V107\% >31.5\% and treated volume TV-V110\% $>4.37 \%$ were associated with higher incidence of acute radiation dermatitis. These reported values are significantly higher than $0 \%$ of $\mathrm{V} 107 \%$ for PTV in our patients treated with FIF w/ 2RP. According to Vicini et al (6), the breast volume V105\% and V110\% are significantly associated with increasing skin toxicities. Using 3D treatment planning and intensity modulation with an MLCs technique, the authors found a significant association between median breast volume V105\% of $11 \%$ and increasing skin toxicities. In our study, using the FIF w/ 2RP method, the mean V105\% for breast volume was reduced to $0.1 \%$.

Considering the reported association between high-dose range and skin toxicities, lowering high-dose areas is likely to reduce the occurrence of radiation dermatitis and its severity. In view of the comparison of the FIF w/ 2RP method with the $\mathrm{W}$ and FIF w/o 2RP methods and literature reports, our method proved to reduce high-dose areas, notably V105\% of the target and doses at OARs. Therefore, the FIF w/ 2RP method is expected to diminish skin toxicities.

The awareness of appearance and timing of skin toxicities is crucial to ensure an effective management of patients. The acute phase of radiation dermatitis typically occurs within 30 to 90 days of RT treatment (9). Our study evaluated the association between the time course of RT and skin toxicity grade. Although the FIF w/ 2RP method had a less significant impact on skin toxicity grade by the end of the RT treatment compared with that of the other two irradiation methods, it had a substantial impact during the 3rd and 4th weeks. This is particularly beneficial for the patient, since the appearance of radiation dermatitis can lead to treatment interruptions or cessation (23).

To further assess the impact of the FIF w/ 2RP method on skin toxicities, the maximum skin toxicity grade during the 5 weeks of RT treatment was examined for the three irradiation methods used, in addition to the skin toxicity grades reported in the literature. Tortorelli et al (8) reported, for patients who underwent irradiation with conventional fractionation followed by an electron tumor bed boost, the maximum acute toxicity during or after completion of RT using the RTOG Acute Morbidity Scale. Grades 1, 2 and 3 acute skin toxicities were registered in $45.2,42$ and $13 \%$ of patients, respectively. Borm et al (5) evaluated 255 patients treated with tangential 3D-CRT and prescribed doses of 50.4 or 50.0 Gy, followed by a sequential boost to the tumor bed for $92.5 \%$ of the patients, using the CTCAE V.4.0 scale. By the end of the treatment, skin toxicity grade 1 was observed in $42.4 \%$ of patients, grade 2 in $55.7 \%$ and grade 3 in $2 \%$. Wright et al (15) reported that, in patients treated only with conventionally fractionated irradiation, $42 \%$ developed CTCAE grade $0-1$ skin toxicity and 58\% developed grade 2-3. Vicini et al (6) reported that, using the MLCs IMRT technique, a prescribed dose of 45 Gy followed by a supplemental boost to the tumor bed of $16 \mathrm{~Gy}$, a total of $56 \%$ of patients experienced RTOG grade 0 or 1 acute skin toxicity, while $43 \%$ experienced grade 2 acute skin toxicity and only $1 \%$ experienced grade 3 toxicity. In our study, compared with the observations in the $\mathrm{W}$ and FIF w/o 2RP methods, for patients treated with the FIF w/ 2RP method, the skin toxicity was reduced, since $5.5 \%$ experienced grade $0,91.3 \%$ grade 1 , $3.2 \%$ grade 2 and none grade 3 . This clinical outcome supports that FIF w/ 2RP is a useful method to reduce both high-dose areas and acute skin toxicity.

Previous studies identified predictive factors for acute skin toxicities and categorized them into patient-related factors and treatment procedure-related factors. Our study, through the evaluation of association between these factors and skin toxicity, demonstrated that the irradiation methods have a greater impact on skin toxicity compared with the patients' baseline characteristics. Breast size was reported as a predictive factor for acute skin toxicity (9). In our study, the correlations between breast volume and skin toxicity grade 
throughout RT treatment, although markedly weak, they were significant. Separation, PTV volume and weight also had a markedly weak, but significant impact on the skin toxicity maximum grade during the 5 weeks of RT treatment, whereas tumor site had significant impact at weeks 3 and 4. Overall, the effect of the patients' baseline characteristics on skin toxicity was limited, while the irradiation methods effect, particularly that of the FIF w/ 2RP method, was comparatively substantial. Associations with the skin toxicity grade were significant when comparing the FIF w/ 2RP method vs. the W or the FIF w/o 2RP methods, indicating that a significant decrease in skin toxicity grade might be expected when using the FIF w/ 2RP method. The utmost benefit of our method can be perceptible during the RT treatment at weeks 3 and 4, as skin toxicity grade is significantly improved compared with that of the $\mathrm{W}$ and to the FIF w/o 2RP methods. This decline in skin toxicities during the treatment course minimizes risks of interruption or cessation and provides patient comfort.

Assessment of skin toxicities is of a subjective nature; however, in our institution, the skin toxicity grade was evaluated by a single experienced radiation oncologist with a standardized approach, which reduces potential variations and ensures a reproductible quantification. The FIF w/ 2RP method is a clinically practical and achievable method; nevertheless, it is more time consuming than conventional irradiation methods. Furthermore, the impact of this method is more considerable in patients with small-sized breasts; therefore, this aspect should be taken into consideration when selecting the appropriate irradiation method.

In conclusion, our study confirmed that the FIF w/ 2RP method decreased the high-dose range V105\% of the target to $0 \%$, while maintaining a homogeneous dose distribution across the breast tissue. This decrease in high-dose range was in conjunction with a decrease in the occurrence and grade of skin adverse events. Therefore, the FIF w/ 2RP could be advised as an optimal method in clinical practice for patients with early stage breast cancer.

\section{Acknowledgements}

Not applicable.

\section{Funding}

No funding was received.

\section{Availability of data and materials}

The datasets used and/or analyzed during the current study are available from the corresponding author on reasonable request.

\section{Authors' contributions}

NT and MK conceived and designed the study, processed the data and wrote the manuscript. MK and HIs assessed the authenticity of the raw data. KKa, SS, KW, KY, NK, TO and $\mathrm{HIh}$ were involved in the clinical studies and in the collection and assembly of the data. HIs, MB, KH, KKo, KS, SK, AK and JA were involved in data analysis and interpretation. NT, MK,
HIs, AK, SK and JA edited the article. All authors have read and approved the final version of the manuscript.

\section{Ethics approval and consent to participate}

The present study was approved by the Ethics Committee of Okayama University Graduate School of Medicine, Dentistry and Pharmaceutical Sciences, and Okayama University Hospital (approval no. 1907-027). Patients provided written informed consent for undergoing RT and using their anonymous data for scientific studies. The institutional informed consent forms for treatment included consent for the use of patient data and materials for research purposes.

\section{Patient consent for publication}

Not applicable.

\section{Competing interests}

The authors declare that they have no competing interests.

\section{References}

1. Clarke M, Collins R, Darby S, Davies C, Elphinstone P, Evans V, Godwin J, Gray R, Hicks C, James S, et al: Effects of radiotherapy and of differences in the extent of surgery for early breast cancer on local recurrence and 15-year survival: An overview of the randomised trials. Lancet 366: 2087-2106, 2005.

2. Early Breast Cancer Trialists' Collaborative Group (EBCTCG), Darby S, McGale P, Correa C, Taylor C, Arriagada R, Clarke M, Cutter D, Davies C, Ewertz M, et al: Effect of radiotherapy after breast-conserving surgery on 10-year recurrence and 15-year breast cancer death: Meta-analysis of individual patient data for 10,801 women in 17 randomised trials. Lancet 378: 1707-1716, 2011.

3. Keenan LG, Lavan N, Dunne M and McArdle O: Modifiable risk factors for acute skin toxicity in adjuvant breast radiotherapy: Dosimetric analysis and review of the literature. Med Dosim 44: $51-55,2019$

4. Schnur JB, Ouellette SC, Dilorenzo TA, Green S and Montgomery GH: A qualitative analysis of acute skin toxicity among breast cancer radiotherapy patients. Psychooncology 20: 260-268, 2011.

5. Borm KJ, Loos M, Oechsner M, Mayinger MC, Paepke D, Kiechle MB, Combs SE and Duma MN: Acute radiodermatitis in modern adjuvant 3D conformal radiotherapy for breast cancer-the impact of dose distribution and patient related factors. Radiat Oncol 13: 218, 2018

6. Vicini FA, Sharpe M, Kestin L, Martinez A, Mitchell CK, Wallace MF, Matter R and Wong J: Optimizing breast cancer treatment efficacy with intensity-modulated radiotherapy. Int J Radiat Oncol Biol Phys 54: 1336-1344, 2002.

7. Chen MF, Chen WC, Lai CH, Hung $\mathrm{CH}$, Liu $\mathrm{KC}$ and Cheng $\mathrm{YH}$ : Predictive factors of radiation-induced skin toxicity in breast cancer patients. BMC Cancer 10: 508, 2010.

8. Tortorelli G, Di Murro L, Barbarino R, Cicchetti S, di Cristino D, Falco MD, Fedele D, Ingrosso G, Janniello D, Morelli P, et al: Standard or hypofractionated radiotherapy in the postoperative treatment of breast cancer: A retrospective analysis of acute skin toxicity and dose inhomogeneities. BMC Cancer 13: 230, 2013.

9. Kole AJ, Kole L and Moran MS: Acute radiation dermatitis in breast cancer patients: Challenges and solutions. Breast Cancer (Dove Med Press) 9: 313-323, 2017.

10. ClinicalTrials: Radiation Therapy Oncology Group: NRG oncology RTOG 1005. A phase III trial of accelerated whole breast irradiation with hypofractionation plus concurrent boost versus standard whole breast irradiation plus sequential boost for early-stage breast cancer. Available from: https://www.rtog. org/ClinicalTrials/ProtocolTable/StudyDetails.aspx?action=ope nFile \&FileID $=9366$. 
11. RadiationTherapyOncologyGroup:BreastCancerAtlas.Available from: https://www.rtog.org/CoreLab/ContouringAtlases/ BreastCancerAtlas.aspx.

12. National Cancer Institute: Common Terminology Criteria for Adverse Events v3.0 (CTCAE). Available from: https://ctep. cancer.gov/protocolDevelopment/electronic_applications/docs/ ctcaev3.pdf.

13. International Classification of Diseases for Oncology. Available from: https://apps.who.int/iris/bitstream/handle/10665/96612/97 89241548496_eng.pdf.

14. Sheng Y, Li T, Yoo S, Yin FF, Blitzblau R, Horton JK, Ge Y and Wu QJ: Automatic planning of whole breast radiation therapy using machine learning models. Front Oncol 9: 750, 2019.

15. Wright JL, Takita C, Reis IM, Zhao W, Lee E, Nelson OL and $\mathrm{Hu}$ JJ: Prospective evaluation of radiation-induced skin toxicity in a race/ethnically diverse breast cancer population. Cancer Med 5: 454-464, 2016.

16. Giri UK, Sarkar B, Jassal K, Munshi A, Ganesh T, Mohanti B and Pradhan A: Left-sided breast radiotherapy after conservative surgery: Comparison of techniques between volumetric modulated arc therapy, forward-planning intensity-modulated radiotherapy and conventional technique. J Radiother Pract 16: 1-8, 2016.

17. Osei E, Darko J, Fleck A, White J, Kiciak A, Redekop R and Gopaul D: Dosimetric evaluation of whole-breast radiation therapy: Clinical experience. Med Dosim 40: 355-365, 2015.

18. Duma MN, Herr AC, Borm KJ, Trott KR, Molls M, Oechsner M and Combs SE: Tangential field radiotherapy for breast cancer-the dose to the heart and heart subvolumes: What structures must be contoured in future clinical trials? Front Oncol 7: 130, 2017.
19. Beaton L, Bergman A, Nichol A, Aparicio M, Wong G, Gondara L, Speers C, Weir L, Davis M and Tyldesley S: Cardiac death after breast radiotherapy and the QUANTEC cardiac guidelines. Clin Transl Radiat Oncol 19: 39-45, 2019.

20. Chung Y, Yoon HI, Kim YB, Ahn SK, Keum KC and Suh CO: Radiation pneumonitis in breast cancer patients who received radiotherapy using the partially wide tangent technique after breast conserving surgery. J Breast Cancer 15: 337-343, 2012

21. Yang DS, Lee JA, Yoon WS, Chung SY, Lee S, Kim CY, Park YJ and Son GS: Whole breast irradiation for small-sized breasts after conserving surgery: Is the field-in-field technique optimal? Breast Cancer 21: 162-169, 2014.

22. Morganti AG, Cilla $S$, de Gaetano A, Panunzi $S$, Digesù $C$, Macchia G, Massaccesi M, Deodato F, Ferrandina G, Cellini N, et al: Forward planned intensity modulated radiotherapy (IMRT) for whole breast postoperative radiotherapy. Is it useful? When? J Appl Clin Med Phys 12: 3451, 2011.

23. Drost L, Li N, Vesprini D, Sangha A, Lee J, Leung E, Rakovitch E, Yee C, Chow E and Ruschin M: Prospective study of breast radiation dermatitis. Clin Breast Cancer 18: e789-e795, 2018.

(i) (3) This work is licensed under a Creative Commons Attribution-NonCommercial-NoDerivatives 4.0 International (CC BY-NC-ND 4.0) License. 